\title{
Mass Spectrometry in an Undergraduate Setting: Clarkson University
}

Katherine M Beglinger, Kelly L Wormwood, Jarrod W Mattingly, Coleman R Larlee, Bethany M. Wawro, Blake Woods and Costel C Darie*

Biochemistry and Proteomics Group, Department of Chemistry and Biomolecular Science, Clarkson University, USA

\begin{abstract}
Mass Spectrometry (MS) is a powerful method used in life sciences that has many applications in various projects. Although very powerful, MS is still not taught in undergraduate settings. Within roughly 3,000 colleges within the USA, MS is taught only in about 300-400 colleges. Clarkson University is one of them. Here we discuss MS and its applications at Clarkson University, with a focus on the Biochemistry I lecture, Biochemistry and Biotechnology laboratory and on Protein Chemistry and Proteomics lecture-laboratory hybrid. Specific examples of the use of MS in academia (basic research), pharmaceutical or biotechnology industry, and in internships in various settings is also discussed.
\end{abstract}

Keywords: Biochemistry; Mass spectrometry; Proteomics; Undergraduate education

\section{Introduction}

Mass Spectrometry (MS) is a powerful method used in life sciences that has many applications in various projects [1-6]. Whether to identify and characterize proteins (protein post-translational modifications, protein-protein interaction), small molecules, lipids, or glycoproteins, the use of MS led to the establishment of new fields such as proteomics for the investigation of proteomes, metabolomics for investigation of metabolomes, lipidomics for the investigation of lipids or glycomics for the identification of oligosaccharides in the glycoproteins [7-11]. Although very powerful, MS is still not standardly taught in undergraduate settings. Yet, in biochemistry textbooks, examples of MS spectra are shown for identification of peptides, proteins, glycolipids, RNA and lipids. Within roughly 3,000 colleges within the USA, MS is taught only in about 300-400 colleges. Clarkson University is one of them. Here we discuss MS and its applications at Clarkson University, with a focus on the Biochemistry I lecture, Biochemistry and Biotechnology laboratory and on Protein Chemistry and Proteomics lecture-laboratory hybrid. Specific examples of the use of MS in academia (basic research), pharmaceutical or biotechnology industry, and in internships in various settings is also discussed.

\section{Biochemistry lecture}

In the Biochemistry course at Clarkson University, students are introduced to simple biochemical structures and characteristics. The first part of the course introduces students to amino acids by learning their structures and physio-chemical properties. They then learn about peptidic bonds and are eventually introduced to an overall protein structure. Once students have a basic understanding of protein structure, they learn about post-translational modifications as well as protein-protein interactions.

Once they have a basic understanding of protein chemistry the students are introduced to biochemical principles of protein purification. They become familiar with the theory behind ionexchange chromatography, size exclusion chromatography, and affinity chromatography. They also learn the theory behind identification techniques such as Western blotting, and gel electrophoresis (SDSPAGE, and 2D-PAGE, etc). Specific PAGE methods such as Differential electrophoresis (DIGE), Blue Native PAGE (BN-PAGE), Colorless Native PAGE (CN-PAGE), Tricine-PAGE, SDS-PAGE under nonreducing conditions (NR-PAGE), as well as a combination of $\mathrm{BN}$ PAGE with SDS-PAGE or Tricine-PAGE, CN-PAGE with SDS-PAGE ot Tricine-PAGE, CN-PAGE with BN-PAGE [12-16]. The students are also introduced to sequencing identification techniques including Sanger and Edman sequencing as well as enzymatic digestion of proteins followed by sequencing.

One unique characteristic of this Biochemistry class is the introduction of MS, which is a much more comprehensive technique used to identify complex protein sequences. Students are taught the full proteomic approach beginning with fractionation, digestion, MS and database search, followed by identification and characterization of proteins. Once they are familiar with this they are introduced to Matrix Assisted Laser Desorption Ionization (MALDI) and Electro Spray Ionization (ESI) based MS. They learn the differences between each method and how they can be applied to protein identification. When learning about MALDI they are introduced to peptide mass fingerprinting whereas with ESI they learn about ESI-MS and ESI-MS/ MS with a live demo of the technique.

Another very unique characteristic to Clarkson's Biochemistry class is that students are taught de novo sequencing and this material is also included in exams. They are taught how to determine the charge and mass of peptides and of high mass proteins that are analyzed by ESI-MS. Students in this class are also taught about the combination of Liquid Chromatography (LC) with MS. Here they learn about LCtandem-MS (LC-MS/MS) which can be used to analyze many peptides (and inherently proteins) over time using a linear gradient elution using reversed phase column and HPLC (RP-LC).

\section{Biochemistry/Biotechnology laboratory}

Clarkson University also has a lab course devoted to learning techniques commonly used in biotechnology. These include protein quantification, protein purification using chromatography, western blotting, and protein identification. It also provides students with the opportunity to learn how MS can be applied to fields of biotechnology. This lab is a nice setting that reinforces the theoretical knowledge implemented in the Biochemistry class into practical, experimental settings. It also allows the students to apply the theory they have learned in the biochemistry class into an independent project.

Students begin by performing an albumin purification using various chromatography techniques. Once the albumin is isolated and purified it is quantified using various methods including the Bradford

*Corresponding author: Costel C Darie, Biochemistry and Proteomics Group Department of Chemistry and Biomolecular Science, Clarkson University, 8 Clarkson Avenue, Potsdam, NY, 13699-5810, USA, Tel: 315-268-7763; Fax: 315268-6610; E-mail: cdarie@clarkson.edu

Received October 03, 2013; Accepted October 21, 2013; Published October 28, 2013

Citation: Beglinger KM, Wormwood KL, Mattingly JW, Larlee CR, Woods B, et al. (2013) Mass Spectrometry in an Undergraduate Setting: Clarkson University. Mod Chem appl 1: 112. doi:10.4172/2329-6798.1000112

Copyright: (c) 2013 Beglinger KM, et al. This is an open-access article distributed under the terms of the Creative Commons Attribution License, which permits unrestricted use, distribution, and reproduction in any medium, provided the original author and source are credited. 
assay and the Lowry method. The sample is then run using an SDSPAGE gel. Students are then taught about different staining techniques including $\mathrm{Zn}$ transient, negative staining as well as a permanent, positive Coomasie blue staining. They then isolate the albumin band and perform a complete proteomic experiment on it, which includes a digestion with trypsin and LC-MS/MS analysis. The proteins are then identified using online database searches.

Students are also introduced to MS by doing a series of direct infusion experiments of peptide fragments using ESI-MS (MALDIMS is also performed, but only in silico, since the available MALDI systems are currently down). The data is then provided and students are able to identify, (using specialized software like Masslynx), the peptide and then protein sequence that was investigated. They are then able to identify the charge, mass, and peptide sequence using de novo sequencing and other bioinformatics tools. Once students have this information, they are able to identify the protein in question based on the mass of the peptide as well as a post-translational modification. They are also taught how to identify a peptide mass fingerprint using various parameters. This shows students the influence of the database search parameters as well as the accuracy of the instrument on which the results are obtained. These experiments reinforce the direct infusion and sequencing experiments taught in the Biochemistry class and gives further detailed analysis of a post-translational modification (acetylation). The full proteomics experiment is also completed. Once the protein/proteins is/are identified in a database search, the students are also required to verify the validity of the results by inspecting the raw data that produced the initial results.

An important requirement for the students in this lab is that once they learn how to use the biochemical principles and techniques, they are required to apply them to an independent project. Specifically, they have to design, propose, budget, conduct and perform a project and then defend its outcomes. This is a particularly good experience for the students. Not surprisingly, the students incorporate MS in their independent project. One example of an experiment that was performed was seeing how effective various contact solutions were at removing proteins from lenses. This utilized quantification methods which would show which solutions removed the highest amount of protein. The protein samples were then separated by SDS-PAGE to identify protein bands that could indicate the presence of proteins from tears. A digestion with trypsin followed by LC-MS/MS analysis, data processing and database search led to identification of proteins from tears. Other experiments include the investigation of protein contents of various meats, yogurts, protein bars, protein shakes, beer or even enzymes from different laundry detergents.

MS was not only used to identify proteins, but also for analysis of small molecules, as well. Among them were identification and quantification of caffeine levels in coffee, tea and some soda drinks, the effects of a temperature gradient in the composition of tea and of the concentration of theine, theophylline and theobromine when making the tea. Lastly, determination of the levels of tannins in various kinds of wine was also investigated by MS.

\section{Protein chemistry and proteomics hybrid lecture-lab}

In addition to training in MS and proteomics, the students also have an extra option: to gain an in-depth understanding of MS and of various mass spectrometers. Although this course is a graduate level course, the undergraduate students interested in working directly in MS and proteomics fields in a graduate school or in the biotechnology or pharmaceutical companies can gain extensive experience in the broad fields of MS. Specifically, the students can learn about the three main components of a mass spectrometer (ion source, mass analyzer and detector), about the mechanism of ionization by MALDI and ESI, about mass analyzers and combination of multiple analyzers and how they can be combined and used for various applications. The students also learn about MS-based analysis of peptides, proteins and small molecules, with a focus on proteomics and its applications on various fields (i.e. clinical or basic research). The students also learn about PTMs (i.e. phosphorylation, acetylation, glycosylation, etc.) and about particular strategies to monitor them. Emphasis on a new field, stable and transient protein-protein interactions is also observed. Overall, the students do receive good training in MS and proteomics, training demanded and sometimes required by most of the biotechnology and pharmaceutical companies, as well as by graduate school committees.

\section{Biochemistry and proteomics group laboratory}

Undergraduate students also gain extensive experience through paid or volunteer summer internships/research experience, or through the full academic year, either though directed research in biochemistry, undergraduate thesis or through the honors program. Worth mentioning it is that these students are also very productive researchers, not only with poster presentations at local, regional and national or international meetings, but also with authorship in research publications [15-20].

\section{Industry applications}

MS is an important method in several different industrial fields, including the biotechnology and pharmaceutical industry. These companies use the same techniques that are taught during the biochemistry class and biotechnology lab to characterize their products. During research and development, these types of techniques, specifically MS, allow pharmaceutical companies to identify if an antibody that may be used for a drug has been successfully made. MS also provides companies with a technique that can be used to identify modifications on proteins and other biochemical molecules that may contribute to the success of a drug. Some recent drugs are proteins or modified proteins. For example, just listing proteins, they are produced by molecular biology methods (recombinant technology) and they have to be identified and characterized, both tasks accomplished by MS. Similarly, when antibodies are used as drugs and drug carriers, (and there is a rather extensive list of drugs that are antibody-based drugs), they are also purified and characterized (again by MS) and/or conjugated with other drugs that require characterization.

Pharmaceutical companies are not the only type of industry that utilizes MS. This is a very flexible technique that also allows other molecules such as lipids and small molecules to be analyzed. This can be applied to several other areas including polymer chemistry, oil, and even forensics. In fact, even customs in the airports use MS to detect explosives.

The heavy use of MS in industry makes it important for undergraduates to have a basic knowledge of MS. Clarkson University is one of the few undergraduate institutions that teach MS. These students have a competitive advantage in industry over those who may not have been taught this highly desired technique.

\section{Examples of MS-based undergraduate research/ internship experiences}

\section{Internship in a large pharmaceutical company (Procter and Gamble)}

Katie (BS double major Chemistry and Biomolecular Science): 
"Having a background in biochemistry and mass spectrometry made a huge impact on my internship. My project involved having a thorough understanding of the proteins of the hair. My project utilized skills I learned during Biochemistry/Biotechnology Lab, including protein quantification and full proteomic experiments. Having the background in mass spectrometry allowed me to exceed the expectations that were set for my internship by investigating hair protein sequences using SDS-PAGE and MALDI-MS."

\section{Internship in a biotechnology company (RegeneronPharma- ceuticals)}

Coleman: MS viewed by a chemical engineer. Coleman, a chemical engineering undergraduate gained some experience with MS while working in the Process Sciences department at Regeneron Pharmaceuticals. He also gained experience analyzing MS data in Clarkson University's Proteomics Group. MS viewed by a pre-med student Jarrod (BS Biomolecular Science): "'My experience as an undergraduate using MS at Clarkson University has been positive, instructive, and rewarding. In the classroom setting I was taught multiple times the principles behind what happens in the machine and more importantly how this translates to valuable data. Then, in the laboratory I was able to use these teachings to further analyze experimental data to be published and also identify and confirm results in my own research as well as that of others."

\section{MS viewed by a chemical engineering student}

Bethany (BS Chemical Engineering): '"My experience as an undergraduate with MS has been in Clarkson University's Proteomics Group. As a chemical engineer, MS is not a topic of interest in any classroom, mainly because our curriculum is focused entirely on process engineering using thermodynamic principles. Having started research in this group, and being exposed to work done with MS, has been insightful and gratifying. In addition to taking the Biochemistry class, working in the lab applies classroom learning into a real and rewarding experience. I am learning skills I would not be able to do in my major that I believe will benefit me in my professional goals for years to come."

\section{Summer research in academic settings}

Kelly (BS double major in Chemistry and Biomolecular Science): "My professional experience with MS has been through research in academia. I had the opportunity to attend the ASMS conference which was an absolutely amazing experience. I was able to learn and see what the different MS instruments were capable of and how they were being used in numerous different fields. If we did not have MS in our lab, it would make our research projects and analysis much more difficult."

Blake (high school student, summer intern): "Eggs taste different. Brown egg, white eggs, mass-produced eggs or free-range eggs taste different. Therefore, I biochemically investigated these egg types and tried to identify the difference between them. I ended up presenting the results in a scientific conference. Due to the short term internship, I was not able to investigate the eggs by MS, but I can't wait to do that next year."

\section{Conclusions}

Overall, MS is a tool that almost no academic can afford not to have it. It is a simple, yet very powerful method. The power of MS comes from its applications in almost any particular field, from monitoring the reaction products in organic chemistry to identification of proteins, their modifications and of their interaction partners. Therefore, teaching undergraduate students MS should become one of the highest priorities in any undergraduate institution. From this standpoint, Clarkson students are fortunate. We wish in the near future we could say the same about all US college students.

\section{Acknowledgement}

This work was in part supported by the David A. Walsh '67 Fellowship, awarded to KLW and CCD. This work was also supported in part by Mary Joyce, Robert Matloff, Ken Sandler, Bonhomie Wine Imports, Wolverine Packing Co. and by the SciFund challenge contributors.

\section{References}

1. Darie CC (2013) Investigation of Protein-Protein Interactions by Blue NativePAGE \& Mass Spectrometry. Mod Chem appl 1: e111.

2. Darie CC (2013) Mass spectrometry and its application in life sciences. Aust $J$ Chem 66: 719-720.

3. Alastair Aitken (2005) Identification of post-translational modifications by mass spectrometry. The Proteomics Protocols Handbook 431-437.

4. Ngounou Wetie AG, Sokolowska I, Woods AG, Roy U, Deinhardt K, et al. (2013) Protein-protein interactions: switch from classical methods to proteomics and bioinformatics-based approaches. Cell Mol Life Sci .

5. Ngounou Wetie AG, Sokolowska I, Woods AG, Roy U, Loo JA, et al. (2013) Investigation of stable and transient protein-protein interactions: Past, present, and future. Proteomics 13: 538-557.

6. Guerrera IC, Kleiner O (2005) Applications of mass spectrometry in proteomics. Biosci Rep 25: 71-93.

7. Darie CC, Litscher ES, Wassarman PM (2008) Structure, processing, and polymerization of rainbow trout egg vitelline envelope proteins. Applications of Mass Spectrometry in Life Safety 23-36.

8. Darie CC, Shetty V, Spellman DS, Zhang G, Xu C, et al. Blue Native PAGE and mass spectrometry analysis of the ephrin stimulation-dependent proteinprotein interactions in NG108-EphB2 cells. Applications of Mass Spectrometry in Life Safety 3-22.

9. Florian PE, Macovei A, Lazar C, Milac AL, Sokolowska I, et al. (2013) Characterization of the anti-HBV activity of HLP1-23, a human lactoferrinderived peptide. J Med Virol 85: 780-788.

10. Roy U, Sokolowska I, Woods AG, Darie CC (2012) Structural investigation of tumor differentiation factor. Biotechnol Appl Biochem 59: 445-450.

11. Woods AG, Sokolowska I, Darie CC (2012) Identification of consistent alkylation of cysteine-less peptides in a proteomics experiment. Biochem Biophys Res Commun 419: 305-308.

12. Schägger H, Cramer WA, von Jagow G (1994) Analysis of molecular masses and oligomeric states of protein complexes by blue native electrophoresis and isolation of membrane protein complexes by two-dimensional native electrophoresis. Anal Biochem 217: 220-230.

13. Schägger $H$, von Jagow $G$ (1991) Blue native electrophoresis for isolation of membrane protein complexes in enzymatically active form. Anal Biochem 199: 223-231.

14. Sokolowska I, Gawinowicz MA, Ngounou Wetie AG, Darie CC (2012) Disulfide proteomics for identification of extracellular or secreted proteins. Electrophoresis 33: 2527-2536

15. Sokolowska I, Woods AG, Wagner J, Dorler J, Wormwood K, et al. (2011) Mass spectrometry for proteomics-based investigation of oxidative stress and heat shock proteins. Oxidative Stress: Diagnostics, Prevention, and Therapy 1083 369-411.

16. Woods AG, Sokolowska I, Yakubu R, Butkiewicz M, LaFleur M, et al. (2011) Blue native page and mass spectrometry as an approach for the investigation of stable and transient protein-protein interactions. Oxidative Stress: Diagnostics, Prevention, and Therapy 1083: 341-367.

17. Ngounou Wetie AG, Sokolowska I, Wormwood K, Beglinger K, Michel TM, et al (2013) Mass spectrometry for the detection of potential psychiatric biomarkers Journal of Molecular Psychiatry 1: 8.

18. Petrareanu C, Macovei A, Sokolowska I, Woods AG, Lazar C, et al. (2013) Comparative Proteomics Reveals Novel Components at the Plasma Membrane of Differentiated HepaRG Cells and Different Distribution in Hepatocyte- and Biliary-Like Cells. PLoS One 8: e71859. 
Citation: Beglinger KM, Wormwood KL, Mattingly JW, Larlee CR, Woods B, et al. (2013) Mass Spectrometry in an Undergraduate Setting: Clarkson University. Mod Chem appl 1: 112. doi:10.4172/2329-6798.1000112

19. Sokolowska I, Dorobantu C, Woods AG, Macovei A, Branza-Nichita N, et al. (2012) Proteomic analysis of plasma membranes isolated from undifferentiated and differentiated HepaRG cells. Proteome Sci 10: 47.
20. Woods AG, Ngounou Wetie AG, Sokolowska I, Russell S, Ryan JP, et al. (2013) Mass spectrometry as a tool for studying autism spectrum disorder. J Molecular Psychiatry 1: 6. 